\title{
Phosphorylation by PKA and Cdk5 Mediates the Early Effects of Synapsin III in Neuronal Morphological Maturation
}

\author{
Alessandra Piccini, ${ }^{1}$ Laura E. Perlini, ${ }^{2}$ Laura Cancedda, ${ }^{2}$ Fabio Benfenati, ${ }^{1,2 *}$ and Silvia Giovedi ${ }^{1 *}$ \\ ${ }^{1}$ Department of Experimental Medicine, University of Genova, 16132 Genova, Italy, and ${ }^{2}$ Department of Neuroscience and Brain Technologies, Istituto \\ Italiano di Tecnologia, 16163 Genova, Italy
}

\begin{abstract}
Synapsin III (SynIII) is a neuron-specific phosphoprotein that plays a unique role in neuronal development. SynIII is phosphorylated by cAMP-dependent protein kinase (PKA) at a highly conserved phosphorylation site and by cyclin-dependent kinase-5 (Cdk5) at a newly described site. Although SynIII is known to be involved in axon elongation in vitro, the role of its phosphorylation by PKA and Cdk5 in the modulation of this process is unknown. We expressed either wild-type (WT) or phosphorylation-site mutants of SynIII in primary SynIII knock-out (KO) mouse neurons at early stages of in vitro development. Whereas the neurite elongation phenotype of SynIII KO neurons was fully rescued by the expression of WT SynIII, the expression of nonphosphorylatable and pseudo-phosphorylated PKA mutants was ineffective. Also, the nonphosphorylatable Cdk5 mutant was unable to rescue the neurite elongation phenotype of SynIII KO neurons. By contrast, the pseudo-phosphorylated mutant rescued the delay in neuronal maturation and axonal elongation, revealing a Cdk5dependent regulation of SynIII function. Interestingly, SynIII KO neurons also exhibited decreased survival that was fully rescued by the expression of WT SynIII, but not by its phosphorylation mutants, and was associated with increased activated caspase 3 and altered tropomyosin receptor kinase B isoform expression. These results indicate that PKA and Cdk5 phosphorylation is required for the physiological action of SynIII on axon specification and neurite outgrowth and that the expression of a functional SynIII is crucial for cell survival.
\end{abstract}

Key words: knock-out mice; neurite outgrowth; neuron survival; protein phosphorylation; synapsin III

\section{Significance Statement}

Synapsin III is an atypical member of the synapsin family of synaptic vesicle-associated phosphoproteins that is precociously expressed in neurons and is downregulated afterward. Although experimental evidence suggests a specific role for Synapsin III in neuronal development, the molecular mechanisms are still largely unknown. We found that Synapsin III plays a central role in early stages of neuronal development involving neuronal survival, polarization, and neuritic growth and that these effects are dependent on phosphorylation by cAMP-dependent protein kinase and cyclin-dependent protein kinase- 5 . These results explain the recently described neurodevelopmental defects in the migration and orientation of Synapsin III-depleted cortical neurons and support the potential association of Synapsin III with neurodevelopmental disorders such as schizophrenia.

\section{Introduction}

Synapsins (Syns) are a family of neuron-specific phosphoproteins associated with synaptic vesicles (SVs) playing important roles in synaptic transmission and plasticity (Cesca et al., 2010). In mammals, three SYN genes have been identified ( SYN1, SYN2, and SYN3), and they are subjected to alternative splicing to give

Received April 10, 2015; revised Aug. 13, 2015; accepted Aug. 19, 2015.

Author contributions: L.C., F.B., and S.G. designed research; A.P. and L.E.P. performed research; A.P., L.C., and S.G. analyzed data; A.P., F.B., and S.G. wrote the paper.

This work was supported by Italian Ministry of University and Research Grants PRIN 2010/11 to F.B. and FIRB 2010 "Futuro in Ricerca" to S.G., European Union FP7 Integrating Project "Desire" Grant 602531 and Cure Epilepsy "Advanced Innovator Award" to F.B., Telethon-Italy Grant GGP13033 to F.B., and CARIPLO Foundation Grant 20130879 to L.C. We thank Dr. Hung-Teh Kao (Brown University, Providence, RI) and Dr. Paul Greengard (Rockefeller University, New York) for providing us with the synapsin III mutant mice, and Dr. Silvia Casagrande for assistance in the preparation of primary cultures. rise to more than 10 isoforms (Südhof et al., 1989; Kao et al., 1998; Porton et al., 1999). Syns are composed of conserved and variable protein domains that confer distinct functional properties (Cesca et al., 2010). At presynaptic terminals, Syns play a critical role in regulating SV trafficking between resting, recycling, and readily releasable pools through phosphorylationdependent interactions with actin and SVs (Valtorta et al., 1992; Cesca et al., 2010; Fornasiero et al., 2012; Orenbuch et al., 2012;

The authors declare no competing financial interests.

*F.B. and S.G. contributed equally to this work.

Correspondence should be addressed to Dr. Fabio Benfenati, Department of Neuroscience and Brain Technologies, Fondazione Istituto Italiano di Tecnologia, Via Morego 30, 16163 Genoa, Italy. E-mail: fabio.benfenati@iit.it. DOI:10.1523/JNEUROSCI.1379-15.2015

Copyright $\odot 2015$ the authors $\quad 0270-6474 / 15 / 3513148-12 \$ 15.00 / 0$ 
Orlando et al., 2014; Verstegen et al., 2014). In addition to the well-known effects in the modulation of synaptic transmission, Syns also play a critical role in neuronal development, by regulating neurite outgrowth and synapse formation (Fornasiero et al., 2010; Perlini et al., 2011).

Despite the shared conserved domains and sequence similarities between the three Syns, SynIII has peculiar characteristics that suggest a functional role distinct from the other two Syns. Indeed, SynIII is expressed in nestin-positive neural progenitors, and its constitutive depletion affects adult neurogenesis in the dentate gyrus of the hippocampus by decreasing cell proliferation and enhancing survival of neural progenitors (Kao et al., 2008). In primary neurons, SynIII displays an early and transient expression profile: it is the first Syn isoform to be expressed and, in contrast with the other Syns, it is not confined to synaptic sites (Ferreira et al., 2000). The early expression pattern of SynIII suggests a role in neuronal development. Indeed, both antisense knocked down neurons (Ferreira et al., 2000) and constitutively knock-out (KO) neurons (Feng et al., 2002) display an impaired differentiation and elongation of the axon, with altered growthcone morphology. These effects may contribute to the impaired radial migration and orientation of neocortical pyramidal neurons that we recently showed to occur in constitutive SynIII KO mice or in wild-type (WT) rats in which SynIII was downregulated (Perlini et al., 2015). However, SynIII KO mice only display a mild behavioral phenotype, are not epileptic, and have a preserved complement of SVs (Feng et al., 2002; Porton et al., 2010).

SynIII shares with the other Syn isoforms a highly conserved cAMP-dependent protein kinase (PKA) phosphorylation site $\left(\right.$ Ser $^{9}$ ) (Kao et al., 1999). Phosphorylation of SynI by PKA modulates synapse formation in vitro (Perlini et al., 2011), and phosphorylation of SynII by PKA plays a crucial role in Xenopus spinal neurons, where it stimulates neurite outgrowth both in vitro and in vivo (Kao et al., 2002). Interestingly, we recently identified a novel phosphorylation site $\left(\operatorname{Ser}^{404}\right)$ for cyclin-dependent kinase-5 (Cdk5) in SynIII that plays a specific role in the in vivo regulation of radial migration of pyramidal neurons (Perlini et al., 2015).

In view of the prominent role of SynIII during early neuronal development, we investigated the role of SynIII phosphorylation by PKA and Cdk5 on neuronal survival and morphology. We demonstrated that the presence of a functional SynIII is crucial for neuronal survival, and we identified a specific role for SynIII during the early stages of neurite extension that is dependent on PKA and Cdk5 phosphorylation.

\section{Materials and Methods}

\section{Generation of constructs}

ShRNAs. ShRNA\#1 was designed against the sequence encoding for the C domain of rSynIII as previously described (Elbashir et al., 2001). ShRNA\#2 was designed against the rSynIII B domain coding sequence with the aid of the BLOCK-iT RNAi Express Sofware (Invitrogen). ShRNA specificity for the mRNA of interest was verified by BLAST aligning with a nonredundant database. The ShRNAs were synthesized by Sigma-Aldrich, annealed in vitro, and ligated into pRNAT-U6.3/Hygro (GenScript).

ShRNA sequences are as follows: \#1: TAACCAGAATGAAGTCAGGTTttgatatccgAACCTGACTTCATTCTGGTTA; and \#2: AAGGAGCT AAACAGGCTGGATttgatatccgATCCAGCCTGTTTAGCTCCTT.

SynIIIa. pEGFP-C3-SynIIIa (gift of dr. H-T. Kao) was digested, and the DNA fragment containing SynIIIa was cloned into pCAGGs-IREStdTomato. The obtained construct pCAGGs-rSynIIIa-IRES-tdTomato was used as a template for the generation of Cdk5 and PKA phosphorylation site mutants. The nonphosphorylatable PKA mutant (pCAGGs-
SynIIIaS9A-IRES-tdTomato) was generated with the primers: forward, 5'-CTCCGGAGGCGGCTGGCCGACAGCAGCTTTGTG; and reverse 5'-CACAAAGCTGCTGTCGGCCAGCCGCCTCCGGAG, which allowed replacing the codon TCA encoding for serine ${ }^{9}$ with the codon GCC encoding for alanine. The pseudo-phosphorylated PKA mutant (pCAGGs-SynIIIaS9E-IRES-tdTomato) was generated with the primers: forward, 5' -CTCCGGAGGCGGCTGGAAGACAGCAGCTTTGTG; and reverse, 5'-CACAAAGCTGCTGTCTTCCAGCCGCCTCCGGAG, which allowed replacing the TCA codon with the codon GAA encoding for glutamic acid.

The nonphosphorylatable Cdk5 mutant (pCAGGs-SynIIIaS404AIRES-tdTomato) was generated with the primers: forward, $5^{\prime}$-CCTCGGTGCCCGCCCCCCTGAGACCTTG; and reverse, 5'-CAAGGTCTC AGGGGGGCGGGCACCGAGG, which allowed replacing the codon TCC encoding for serine ${ }^{404}$ with the GCC codon encoding for alanine. The pseudo-phosphorylated Cdk5 mutant (pCAGGs-SynIIIaS404DIRES-tdTomato) was generated with the primers: forward, $5^{\prime}$-CCTCGGTGCCCGACCCCCTGAGACCTTG; and reverse, 5' -CAAGGTCTCA GGGGGTCGGGCACCGAGG, which allowed replacing the TCC codon with the codon GAC encoding for aspartic acid.

Mutagenesis was performed with QuikChangeLightning Site-Direct Mutagenesis Kit (Stratagene). All constructs were verified by sequencing. All enzymes were purchased by Promega, if not otherwise indicated.

\section{Primary cultures of cortical neurons and neural nucleofection}

Sprague Dawley rats and C57BL/6J mice of either sex were from Harlan or Charles River. SynIII KO mice were generated by homologous recombination (Feng et al., 2002) and extensively backcrossed on the C57BL/6J background for over 10 generations. All experiments were performed in accordance with the guidelines established by the European Communities Council (Directive 2010/63/EU of March 4,2014) and were approved by the Italian Ministry of Health.

Primary cultures of dissociated cortical neurons were prepared from C57BL/6J and SynIII KO E17-E18 mouse embryos or from E17-E18 embryos and newborn P0 rats, plated onto poly-D-lysine $(0.1 \mathrm{mg} / \mathrm{ml}$, Sigma-Aldrich)-coated $25 \mathrm{~mm}$ glass coverslips at a density of $6 \times 10^{4}$ cells/coverslip, and coated wells at a density of $1 \times 10^{6}$ cells/well. Cells were maintained in a culture medium consisting of Neurobasal (Invitrogen ) supplemented with B-27 (1:50 v/v, Invitrogen ), Glutamax $(1 \% \mathrm{w} / \mathrm{v}$, Invitrogen ), penicillin-streptomycin ( $1 \%$ Invitrogen ), and kept at $37^{\circ} \mathrm{C}$ in a $5 \% \mathrm{CO}_{2}$ humidified atmosphere. For transfection experiments, neurons $\left(4 \times 10^{6}\right)$ were nucleofected before plating by electroporation with Amaxa basal nucleofector kit for primary neurons (Lonza) with $4 \mu \mathrm{g}$ of plasmid DNA according to the manufacturer's protocol. The average transfection efficiency, based on Tomato/DAPI fluorescence, was $24.0 \pm$ $1.1 \%$ (mean \pm SEM over $n=70$ transfections). Cells were incubated under standard growth conditions and then processed for immunocytochemistry, Western blotting, or viability assay at various stages of development from 1 to $14 \mathrm{~d}$ in vitro (DIV). Under our culture conditions (Neurobasal/B27 medium and low cell density), $\sim 85 \%-90 \%$ of the cortical neurons are glutamatergic (Stichel and Muller, 1991; Hayashi et al., 2003) and cultures are almost glia-free (Brewer et al., 1993) (mean \pm SEM percentage of astrocytes vs total cells: $1.37 \pm 0.14$ at 3 DIV, $1.56 \pm$ 0.15 at 5 DIV, and $3.96 \pm 0.40$ at 7 DIV; mean \pm SEM percentage of microglial cells vs total cells $1.07 \pm 0.11$ at 3 DIV, $0.79 \pm 0.10$ at 5 DIV, and $1.21 \pm 0.20$ at 7 DIV; $n=4)$.

\section{In utero electroporation (IUE)}

Standard IUE was performed as previously described (dal Maschio et al., 2012). Briefly, E17 timed-pregnant Sprague Dawley rats were anesthetized with isoflurane (induction, 3.5\%; surgery, 2.5\%), and the uterine horns were exposed by laparotomy. The day of confirmation of vaginal plug was defined as E0, and the day of birth was defined as P0. The DNA $(1.5 \mu \mathrm{g} / \mu \mathrm{l}$ in water) together with the dye Fast Green $(0.3 \mathrm{mg} / \mathrm{ml}$; SigmaAldrich) was injected (5-6 $\mu$ l) through the uterine wall into one of the lateral ventricles of each embryo by a $30 \mathrm{G}$ needle (Pic indolor). The embryo's head was carefully held between tweezer-type circular electrodes (10 mm diameter; Nepa Gene) wet with PBS while across the uterus wall. For the electroporation protocol, 5 electrical pulses (ampli- 
tude, $50 \mathrm{~V}$; duration, $50 \mathrm{~ms}$; intervals, $150 \mathrm{~ms}$ ) were delivered with a square-wave electroporation generator (CUY21EDIT; Nepa Gene). After electroporation, the uterine horns were returned into the abdominal cavity, and embryos allowed continuing their normal development.

\section{Immunocytochemistry}

Primary cortical neurons were fixed at 1,3 , or 14 DIV with $4 \%$ PFA (Sigma-Aldrich) and 4\% sucrose in PBS, pH 7.4. After several washes in PBS, cells were permeabilized with $0.1 \%$ Triton X-100 in PBS for 5 min and blocked with $0.1 \%$ Triton X-100, 3\% FBS in PBS for 30 min. Samples were incubated with the following primary antibodies diluted in blocking solution for $2 \mathrm{~h}$ at room temperature: rabbit anti- $\beta$ III-tubulin (1:1000, Sigma-Aldrich), mouse pan-axonal neurofilament marker (SMI312, 1:500, Covance), rabbit anti-SynIII (1:800, Synaptic Systems), mouse anti-p35 (1:50, Abcam), rabbit anti-GFAP (1:1000, Millipore), rabbit anti-Ibal (1:300, Wako), and rabbit anti-GABA (1:500, Sigma). Immunostaining was detected using Alexa-488 or -594-conjugated secondary antibodies (1:500, Invitrogen) diluted in blocking solution for $1 \mathrm{~h}$ at room temperature. After several washes in PBS, coverslips were mounted using Prolong Gold antifade reagent (Invitrogen) containing DAPI for nuclear staining. Images were acquired with an Olympus IX-81 microscope with an MT20 Arc/Xe lamp and a $40 \times$ or $60 \times$ objective, using the Excellence RT software (Olympus), and analyzed with ImageJ software, NeuronJ plugin, for minor process and axonal length.

\section{Slice histology and immunostaining}

Slice histology, immunostaining, and image acquisition were performed as previously described with minor modifications (Perlini et al., 2015). P7 brains of C57BL/6J and SynIII KO mice were fixed by transcardial perfusion of $4 \%$ PFA in PBS and then cryopreserved in 30\% sucrose. Brains were frozen, and $80-\mu \mathrm{m}$-thick coronal sections were obtained with a microtome-refrigerator (Microm HM 450 Sliding Microtome equipped with Freezing Unit Microm KS34, Thermo Scientific). Freefloating slices were permeabilized and blocked with PBS containing $0.3 \%$ Triton X-100, 10\% normal goat serum, and $0.2 \%$ BSA. Rabbit anti-active Caspase3 primary antibody (1:300, R\&D Systems) was incubated in PBS containing $0.3 \%$ Triton X-100, 5\% normal goat serum, and $0.1 \%$ BSA. Immunostaining was detected using Alexa-546 fluorescent secondary antibody (1:400, Invitrogen), diluted in PBS containing $0.3 \%$ Triton X-100, 5\% normal goat serum, and $0.1 \%$ BSA. Slices were counterstained with Hoechst $(2.5 \mu \mathrm{g} / \mu \mathrm{l}$; Sigma-Aldrich). Samples were mounted in Vectashield Mounting Medium (Vector Laboratories) and examined microscopically. For analysis of apoptotic cells, images from the stained sections were acquired using an epifluorescence microscope equipped with a $10 \times$ air objective (NA 0.3 ) and the software Neurolucida (MicroBrightField). The whole slice was acquired, and the entire cortical region was analyzed. One slice per animal selected from the same brain region was analyzed. For representative pictures, $80-\mu \mathrm{m}$-thick $Z$-stacks were acquired with a confocal laser-scanning microscope (TCS SP5; Leica Microsystems) equipped with a $10 \times$ immersion objective (NA 0.4), and $Z$-series of confocal images were projected to two-dimensional representations.

\section{Western blotting}

Total cell lysates were obtained from cortical neuronal cultures at various DIV (from 1 to 7). Cells were lysed in lysis buffer ( $150 \mathrm{~mm} \mathrm{NaCl}, 50 \mathrm{~mm}$ Tris, 1 mu EDTA, 1\% Triton X-100) supplemented with 1 mM PMSF/1 mu pepstatin (Sigma-Aldrich). After 10 min of incubation on ice, lysates were collected and clarified by centrifugation $(10 \mathrm{~min}$ at $10,000 \times g$ at $4^{\circ} \mathrm{C}$ ). Subcellular fractions were prepared from 5- to 6-week-old male Sprague Dawley rat forebrain, and SVs were purified through Controlled-Pore Glass chromatography (Huttner et al., 1983). Purified synaptic vesicles (USV) were recovered by high-speed centrifugation and resuspended in $0.3 \mathrm{~m}$ glycine, $5 \mathrm{~mm}$ HEPES, $\mathrm{pH} 7.4$, at a protein concentration of $1 \mathrm{mg} / \mathrm{ml}$. USV-containing endogenous Syns were quantitatively depleted of Syns by dilution ( $10 \mu \mathrm{g}$ of protein $/ \mathrm{ml}$ ) in $0.15 \mathrm{~m}$ glycine, $0.2 \mathrm{M} \mathrm{NaCl}, 2.5 \mathrm{~mm}$ HEPES, pH 7.4, immediately after elution from the column and incubation for $2 \mathrm{~h}$ in ice. After incubation, salt-treated SVs were recovered by high-speed centrifugation and resuspended in $0.3 \mathrm{M}$ glycine, $5 \mathrm{~mm}$ HEPES, $\mathrm{pH} 7.4$, at a protein concentration of $1 \mathrm{mg} / \mathrm{ml}$.
Protein concentration of the samples was determined by the Bradford Assay (Bio-Rad), and equivalent amounts of protein were subjected to SDS-PAGE on $10 \%$ or $14 \%$ polyacrylamide gels and blotted onto nitrocellulose membranes (Whatman). After a brief staining with $0.1 \%$ Ponceau S, blotted membranes were blocked for $1 \mathrm{~h}$ in $5 \%$ milk in Trisbuffered saline (10 mM Tris, $150 \mathrm{~mm} \mathrm{NaCl}, \mathrm{pH}$ 8.0) plus $0.1 \%$ Triton $\mathrm{X}-100$ and incubated overnight at $4^{\circ} \mathrm{C}$ with the following primary antibodies: rabbit anti- $\beta$ III-tubulin (1:8000, Sigma-Aldrich), rabbit antiSynIII (1:1000, Synaptic Systems), mouse anti-Cdk5 (1:1000, Millipore), rabbit anti-p35 (1:1000, Santa Cruz Biotechnology), rabbit antisynaptophysin (1:5000, Synaptic Systems), rabbit anti-tropomyosin receptor kinase B (TrkB) (1:1000, Millipore), rabbit anti-p75 ${ }^{\text {NTR }}$ (1:1000, Abcam), and rabbit anti-caspase3 (1:1000, Cell Signaling Technology). Membranes were washed and incubated for $1 \mathrm{~h}$ at room temperature with peroxidase-conjugated goat anti-mouse (1:3000; Bio-Rad) or antirabbit (1:5000; Bio-Rad) antibodies. Bands were revealed with the ECL chemiluminescence detection system (Thermo Scientific).

\section{MTT reduction assay}

Cell viability was estimated by the MTT reduction assay, an indicator of the mitochondrial activity of living cells. Primary cortical neurons from SynIII KO and C57BL/6J mice were cultured as described above. At 3 and $7 \mathrm{DIV}$, cells were washed and incubated with the MTT solution for $3 \mathrm{~h}$ in incubator at $37^{\circ} \mathrm{C}(1 / 10$ in serum-free culture medium; stock: $5 \mathrm{mg} / \mathrm{ml}$ in PBS; Sigma-Aldrich). The medium was harvested, and MTT crystals were solubilized by adding DMSO (same volume as MTT medium) and incubated $15 \mathrm{~min}$ at dark, on a shaker. Wells were then read at $570 \mathrm{~nm}$ with background subtraction at $620 \mathrm{~nm}$, using a Benchmark Microplate Reader, with Microplate Manager Version 5.1 software (Bio-Rad).

\section{Statistical analysis}

Data with normal distribution were analyzed by one-way ANOVA followed by the Bonferroni's multiple-comparison test or the unpaired Student's $t$ test. The statistical analysis was performed using the GraphPad software (GraphPad Software). Significance level was preset to $p<0.05$. Data are mean \pm SEM.

\section{Results}

\section{Phenotype of SynIII KO and SynIII silenced cortical neurons}

To investigate the role of SynIII in developing neurons, we first analyzed cell morphology in WT and SynIII KO cortical neurons at early neurodevelopmental stages ( 1 and 3 DIV). We performed immunocytochemistry with anti- $\beta$ III-tubulin antibodies to identify the total neuritic tree and SMI312 antibodies directed against pan-axonal neurofilaments to unambiguously and specifically label axons (Zuccaro et al., 2014). We found that SynIII KO neurons at 1 DIV showed a significant decrease in the length of SMI312-negative (dendritic) minor processes and in the total number of ( $\beta$ III-tubulin positive) neurites compared with WT cells (Fig. $1 A, C$ ). Also, the percentage of cells harboring an axon and the axonal length were significantly decreased in SynIII KO neurons (Fig. 1D). However, these phenotypes were completely rescued at 3 DIV when KO neuronal morphology became comparable with that of WT cells (Fig. $1 B-D$ ). These results indicate that SynIII not only is required to foster axonal extension during the first $24 \mathrm{~h}$ after plating (Ferreira et al., 2000; Feng et al., 2002) but also show, for the first time, that SynIII has a specific role in the outgrowth of nonaxonal processes.

Because the constitutive and widespread depletion of SynIII in KO mice could trigger redundancy or compensatory mechanisms by other proteins that may overcome deficits in neuronal development, we acutely downregulated SynIII expression by RNA interference at prenatal or postnatal phases. Postnatal silencing of SynIII was performed in WT cortical neurons, by Amaxa nucleofection before plating with control scrambled ShRNA (ShRNAscr) or SynIII ShRNA\#1 (Perlini et al., 2015). Although the SynIII ShRNA was effective in decreasing the SynIII 
A

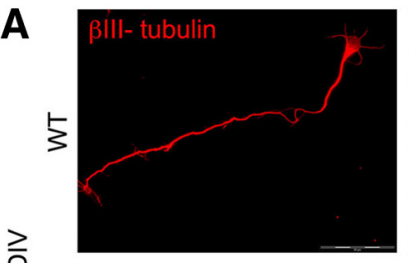

$\stackrel{\text { בे }}{\circ}$

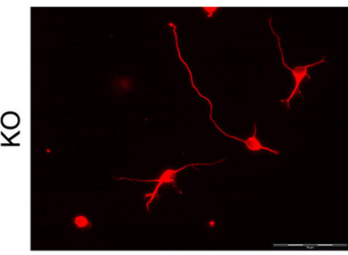

B

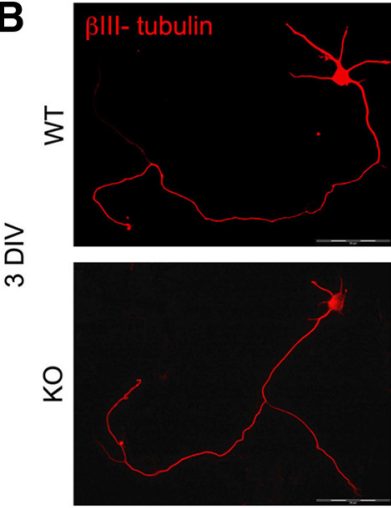

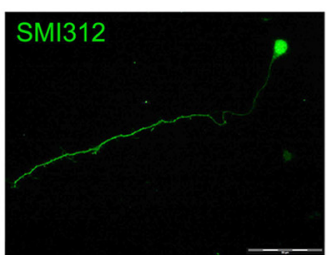
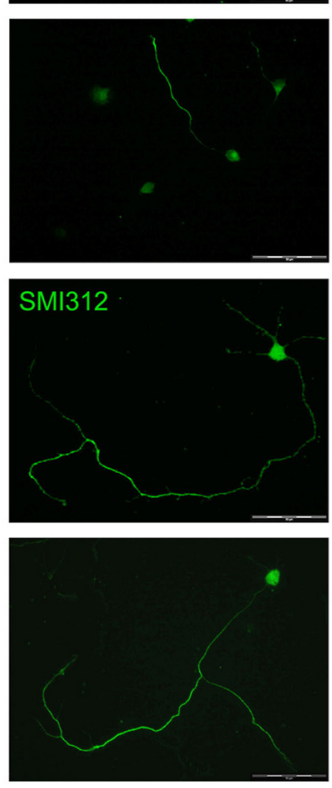
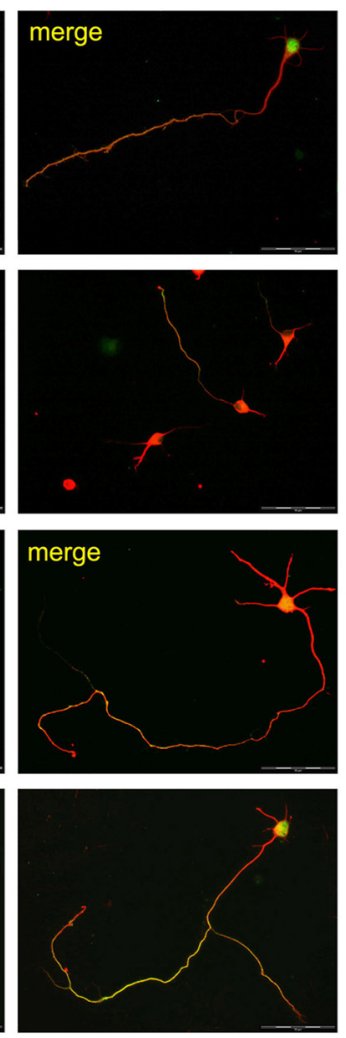
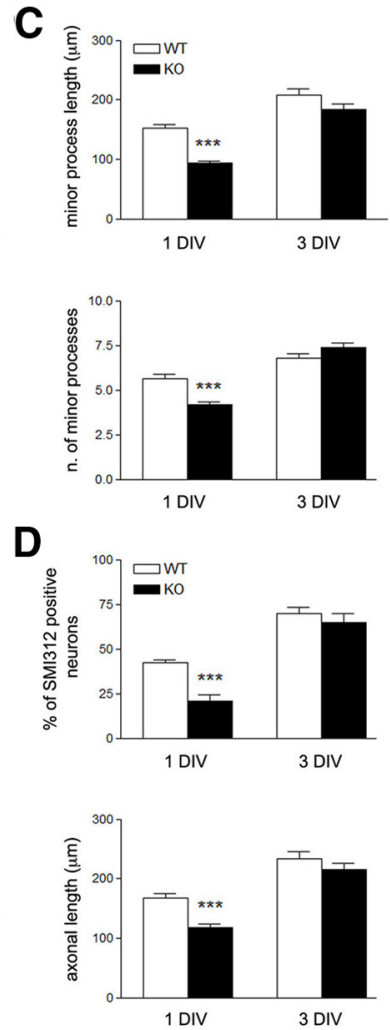

Figure 1. Synlll KO neurons display a delayed maturation at early stages of in vitro development. $\boldsymbol{A}, \boldsymbol{B}$, Representative images of WT and Synlll KO cortical neurons fixed at 1 DIV ( $\boldsymbol{A})$ and 3 DIV ( $\boldsymbol{B})$ and double stained with $\beta$ III-tubulin (red) and SMI312 (green). Scale bar, $50 \mu \mathrm{m}$. C, Morphometric analysis using ImageJ software of the length (top) and number (bottom) of minor processes of WT and SynIII KO cortical neurons at 1 and 3 DIV. D, Quantitative analysis of the percentage of SMI312-positive neurons over total neurons (top) and the axon length of SMI312-positive neurons (bottom) measured in WT and Synlll KO cortical neurons at 1 and 3 DIV. C, D, Data are mean \pm SEM ( $n=108$ WT neurons and $n=121$ K0 neurons at 1 DIV; $n=100$ WT neurons and $n=118$ K0 neurons at 3 DIV from $n=4$ independent experiments). ${ }^{* *} p<0.001$ (unpaired Student's $t$ test).

protein level already at 1 DIV (Fig. $2 A$ ), it did not affect neuronal maturation either at 1 or 3 DIV (Fig. $2 B, C$ ), even in the presence of a slight maturation delay induced by nucleofection. A second SynIII ShRNA gave similar results (ShRNA\#2) (Perlini et al., 2015). Nevertheless, when the downregulation of SynIII was performed during the embryonic period by IUE at E17, the neuronal cultures obtained from the electroporated embryos showed a significant impairment in neuronal development. Indeed, ShRNA\# ${ }^{+}$neurons displayed decreased length and number of SMI312-negative minor processes compared with ShRNAscr ${ }^{+}$ cells at both 1 and 3 DIV (Fig. 2D, left and middle panels, E). The measurement of axonal length showed no differences, but, strikingly, the number of cells displaying axons (SMI312-positive neurons) was dramatically lower in ShRNA\#1 ${ }^{+}$neurons compared with ShRNAscr ${ }^{+}$cells at both 1 and 3 DIV (Fig. 2D, right panels, $F$ ), indicating a strong impairment in axon differentiation. In the few silenced neurons that could extend an axon, however, the axonal length showed no significant differences compared with ShRNAscr ${ }^{+}$cells (Fig. $2 F$ ).

These data allow the establishment of a precise time window for the action of SynIII on neuronal maturation and demonstrate a similar effect of the acute or constitutive depletion of SynIII in neuronal development.

\section{PKA phosphorylation of SynIII is required for early neuronal development}

Phosphorylation by several protein kinases is a crucial functional step that contributes to the modulation of Syn activity. Because SynIII is substrate for PKA, a kinase involved in neuronal sur- vival, neurite outgrowth, and synaptogenesis (Kao et al., 2002; Perlini et al., 2011), we investigated the role of PKA-mediated phosphorylation of SynIII at early stages of development. Toward this aim, we expressed either WT SynIII or two different SynIII mutants on the conserved PKA phosphorylation site $\left(\mathrm{Ser}^{9}\right)$ in cortical SynIII KO neurons via nucleofection. In particular, we used pCAGGs-rSynIIIa-IRES-tdTomato as a template for sitedirected mutagenesis to generate a dephosphomimetic mutant (S9A) in which the nonphosphorylatable alanine replaces serine, and a phosphomimetic mutant (S9E) in which the negatively charged glutamic acid mimics the charge of the phosphate group. The overall expression levels of WT and SynIII mutants, analyzed in SynIII KO neuronal lysates at 3 DIV by Western blotting, were similar and in the same range of the endogenous SynIII in parallel cultures of WT neurons at the same developmental stage (Fig. $3 A$ ), confirming the validity of the constructs and the high transfection efficiency (see Materials and Methods). Then, we performed immunocytochemistry with anti-SynIII antibodies to analyze the expression patterns of the mutant proteins in SynIII KO transfected neurons. WT, S9A, and S9E SynIII were correctly translated and targeted to the cell body and presynaptic nerve terminals with comparable expression patterns (Fig. 3B). Nucleofection caused per se a delay in neuronal maturation leading to a neurite elongation at 3 DIV comparable with that of stage 1 or 2 DIV nontransfected neurons (Fig. 1). Consequently, we analyzed neuronal morphology at 3 DIV in transfected neurons. The morphological analysis showed that the neurite outgrowth phenotype of SynIII KO neurons (transfected with the empty vector) was completely rescued by the expression of WT SynIII. 
A

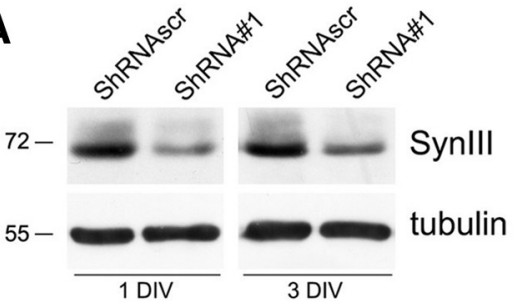

C

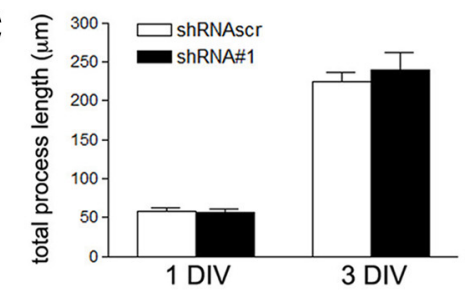

B
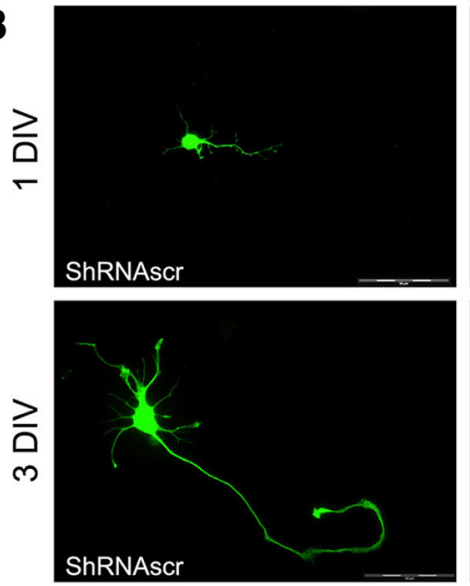
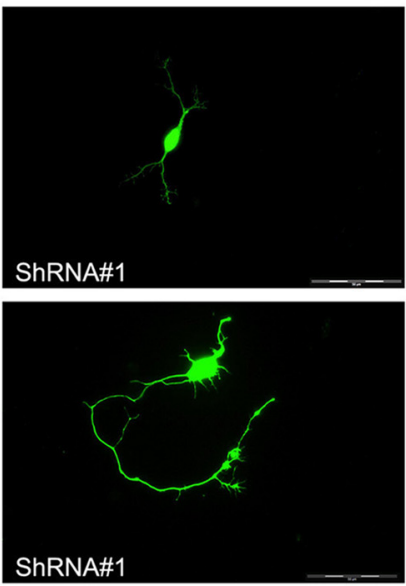
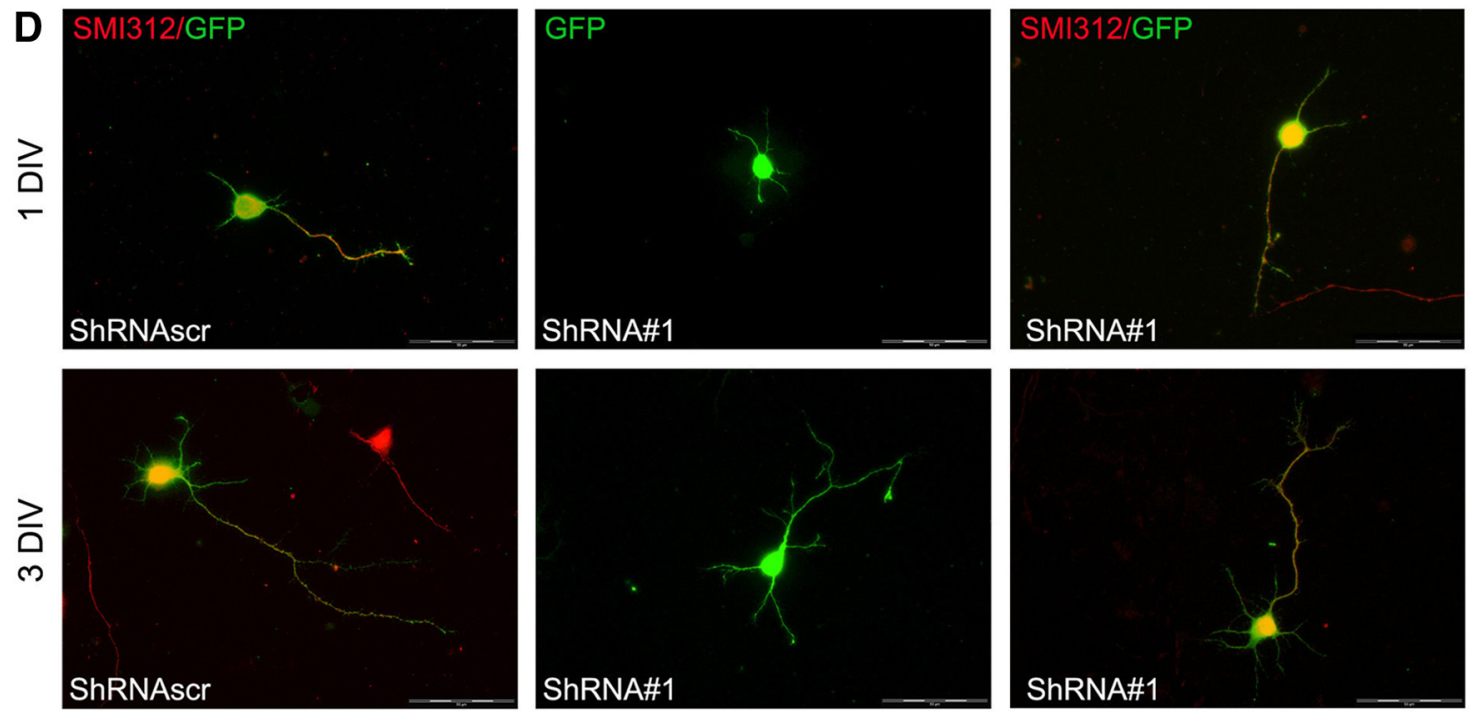

E
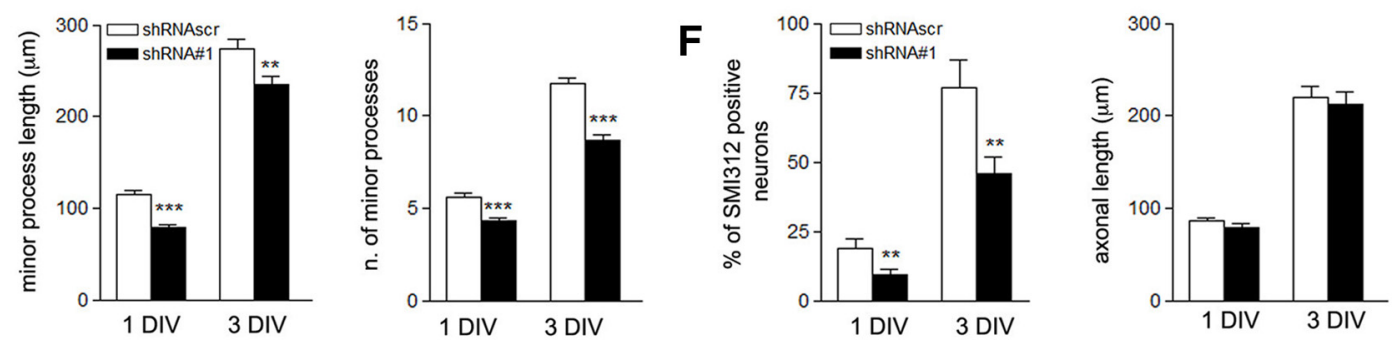

Figure 2. Phenotype of Synlll silenced cortical neurons. $\boldsymbol{A}$, Representative Western blot showing the specificity of Synlll ShRNA\#1 (ShRNA\#1) in downregulating endogenous Synlll levels in transfected WT cortical neurons at 1 and 3 DIV compared with the control scrambled ShRNA (ShRNAscr). Tubulin immunoreactivity was used to control for equal loading. Molecular masses are shown on the left in kDa. B, Representative images of 1 DIV and 3 DIV WT cortical neurons transfected by Amaxa before plating with either ShRNAscr or ShRNA\#1 (green). Scale bar, $50 \mu \mathrm{m}$. C, Quantification of the total process length in the experiments shown in $\boldsymbol{B}$ using Image software. Data are mean \pm SEM $\left(n=90\right.$ ShRNAscr ${ }^{+}$neurons and $n=95$ ShRNA\# ${ }^{+}$neurons at 1 DIV; $n=85$ ShRNAscr ${ }^{+}$ neurons and $n=91$ ShRNA\#1 ${ }^{+}$neurons at 3 DIV, from $n=3$ independent experiments). $p=0.888$ at 1 DIV (unpaired Student's $t$ test). $p=0.593$ at 3 DIV (unpaired Student's $t$ test). $\boldsymbol{D}$, Representative images of primary WT cortical neurons plated after IUE at E17 with either ShRNAscr or ShRNA\#1 (green), fixed at 1 DIV and 3 DIV, and stained for SMI312 (red). Scale bar, $50 \mu \mathrm{m}$. $E$, Quantification of the length and number of minor processes of neurons in the experiments shown in $\boldsymbol{D}$ measured by ImageJ software. ${ }^{* *} p=0.008$ versus the respective ShRNAscr (unpaired Student's $t$ test). ${ }^{* * *} p<0.001$ versus the respective ShRNAscr (unpaired Student's $t$ test). $F$, Quantification of the percentage of SMI312-positive neurons and their axon length. ${ }^{* *} p=0.007$ at 1 DIV (unpaired Student's $t$ test). ${ }^{* *} p=0.008$ at 3 DIV (unpaired Student's $t$ test). $E$, F, Data are mean \pm SEM $\left(n=126\right.$ ShRNAscr ${ }^{+}$neurons and $n=116$ ShRNA\#\# $1^{+}$neurons at 1 DIV; $n=114$

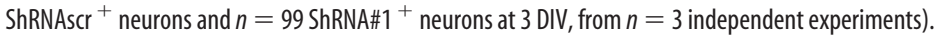

Conversely, expression of either PKA phosphorylation mutant was unable to rescue the KO phenotype in terms of both SMI312negative processes and total number of neurites (Fig. 3C,D). Moreover, the expression of either S9A or S9E SynIII led to a significantly lower percentage of SMI312-positive neurons compared with WT SynIII, with a much stronger effect for S9A SynIII (Fig. 3C,E). However, the few SMI312-positive neurons surpris- ingly showed normal axonal length (Fig. $3 E$ ). In any case, the phenotype of control $\mathrm{KO}$ neurons and of $\mathrm{KO}$ neurons expressing the PKA phosphorylation mutants of SynIII was fully rescued at 7 DIV (mean \pm SEM process length in KO neurons transfected with empty vector, WT SynIII, S9A SynIII, and S9E SynIII was $1160 \pm 56,1165 \pm 62,1168 \pm 60$, and $1164 \pm 56 \mu \mathrm{m}$, respectively; $p>0.05$, one-way ANOVA; $n=10-11$ /experimental 
A

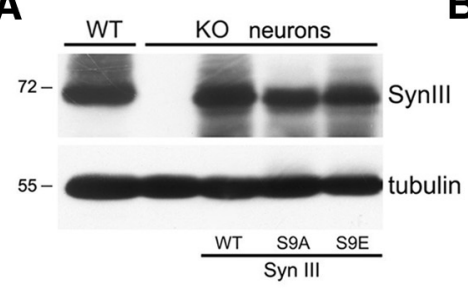

B

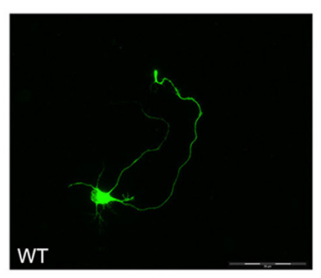

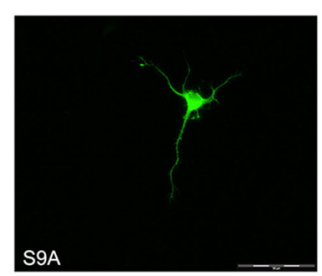
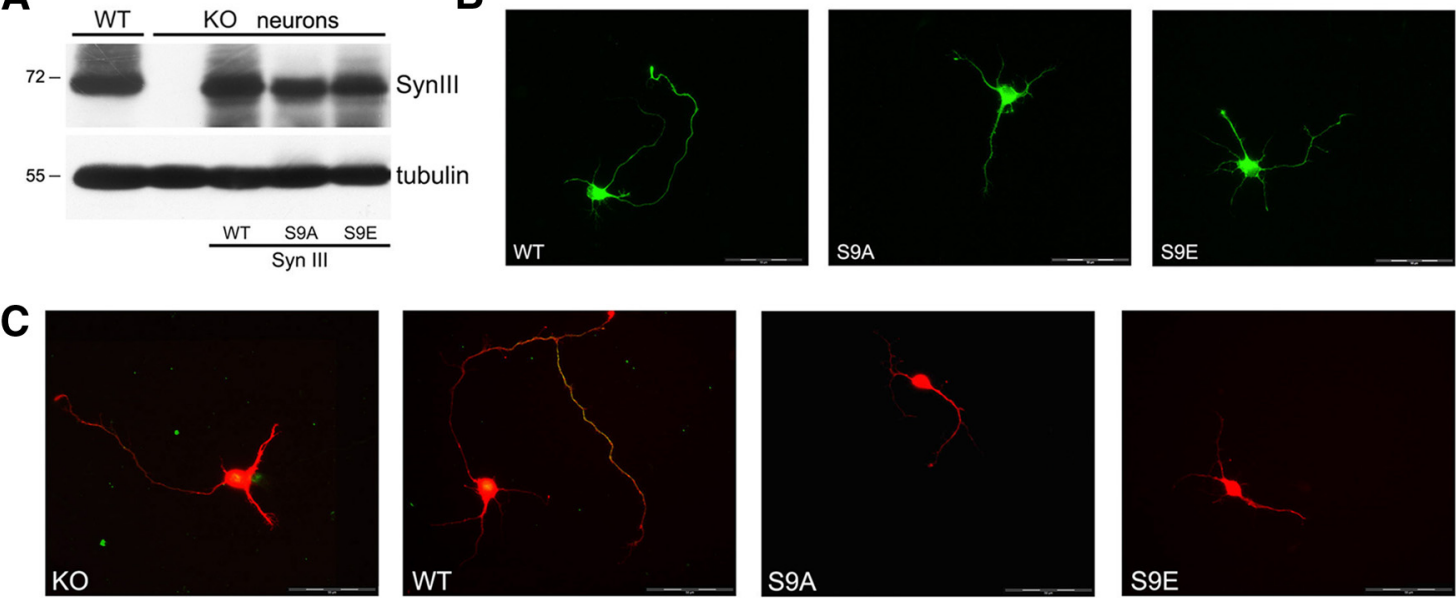

D
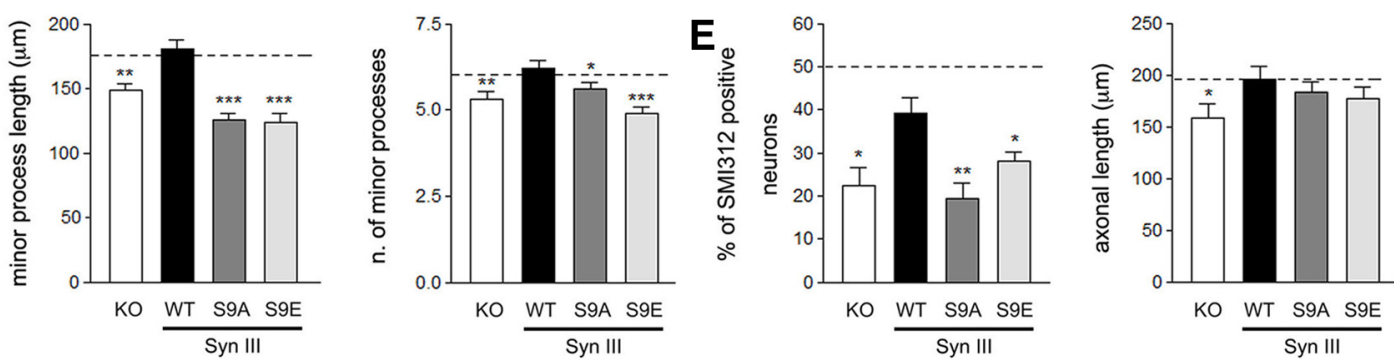

Figure 3. PKA phosphorylation of SynIII is required for early neuronal development. $\boldsymbol{A}$, Representative Western blot of SynIII expression in WT neurons, KO neurons, and KO neurons expressing either WT SynIII or its PKA mutants. Primary cortical neurons from either WT or SynIII KO mice were transfected by Amaxa before plating with the empty pCAGGs-IRES-tdTomato vector (WT and KO neurons). For rescue experiments, SynIII KO neurons were transfected with pCAGGs-IRES-tdTomato vectors encoding WT-SynIII, S9A-SynIII, or S9E-SynIII. Neurons were lysed at 3 DIV and subjected to Western blotting. Tubulin immunoreactivity was used to control for equal loading. Molecular masses of the proteins are on the left in kDa. $\boldsymbol{B}$, Representative images of 3 DIV tdTomato-positive SynIII KO cortical neurons expressing WT-SynIII, S9A-SynIII, or S9E-SynIII. Fixed cells were stained for SynIII (green). WT-Synlll and both mutants showed a similar expression and distribution in all subcellular compartments. Scale bar, $50 \mu \mathrm{m}$. C, Representative images of 3 DIV Synlll K0 cortical neurons expressing tdTomato alone (K0) or with WT-Synlll, S9A-Synlll, or S9E-Synlll (red) and stained for SMI312 (green). Scale bar, $50 \mu \mathrm{m}$. D, Quantification of the length and number of minor processes of neurons in the experiments shown in $\boldsymbol{C}$ measured by ImageJ software. $\boldsymbol{E}$, Quantification of the percentage of SMI312-positive neurons and their axon length. Data are mean \pm SEM ( $n=142 \mathrm{KO}$ neurons, $n=130 \mathrm{WT}$-Synlll neurons, $n=154$ S9A-SynllI neurons, and $n=$ 112 S9E-SynIII neurons at 3 DIV, from $n=4$ independent experiments). ${ }^{*} p<0.05$ versus WT (one-way ANOVA followed by the Bonferroni's multiple-comparison test). ${ }^{* *} p<0.01$ versus WT (one-way ANOVA followed by the Bonferroni's multiple-comparison test). ${ }^{* * *} p<0.001$ versus WT (one-way ANOVA followed by the Bonferroni's multiple-comparison test). Dashed lines indicate the mean values of WT cortical neurons transfected with empty pCAGGs-IRES-tdTomato at 3 DIV included for comparison.

group from 3 independent preparations). Together, these data indicate that the presence of SynIII and PKA phosphorylation/ dephosphorylation cycles are both necessary for early development of neuronal processes and axon specification/outgrowth. By contrast, at later stages, other mechanisms allow neurons to catch up a physiological growth.

\section{A novel Cdk5 phosphorylation site on SynIII regulates its functions}

It has been previously reported that SynI, the main member of the Syn family, is phosphorylated by Cdk5, and this phosphorylation homeostatically regulates the transition of SVs between resting and recycling pools in mature terminals (Jovanovic et al., 1996; Matsubara et al., 1996; Verstegen et al., 2014). We have recently described that Cdk5 physically interacts with and phosphorylates SynIII at a unique phosphorylation site $\left(\mathrm{Ser}^{404}\right)$ that is crucial for the regulation of radial migration of pyramidal neurons in the developing neocortex (Perlini et al., 2015). SynIII and Cdk5 are coexpressed at perinatal ages in the cortex, but thereafter their expression patterns diverge (Ferreira et al., 2000; Odajima et al., 2011; Perlini et al., 2015). Before addressing the role of Cdk5 phosphorylation of SynIII, we checked the distribution of SynIII and p35, the neurospecific Cdk5 activator, in WT cortical neurons at 3 and 14 DIV. During early in vitro development (3 DIV), the two proteins shared an almost total colocalization at the level of the cell body and growth cones, confirming the extrasynaptic localization of SynIII (Ferreira et al., 2000). However, after maturation and synaptogenesis (14 DIV), the two proteins colocalized only at synaptic boutons (Fig. 4A). Subcellular distribution of SynIII and Cdk5/p35 in rat forebrain fractions showed that SynIII and Cdk5/p35 were coenriched in synaptic terminal (LP2 and SG4) and SV fractions identified by the specific SV marker synaptophysin (Fig. 4B). These results confirm that SynIII and Cdk5/p35 colocalize both in developing and mature neurons.

To evaluate the functional role of $\mathrm{Cdk} 5$ phosphorylation of SynIII in the in vitro neuronal development, we took advantage of dephosphomimetic and phosphomimetic Cdk5 mutants of SynIII (Perlini et al., 2015), where Ser ${ }^{404}$ was replaced with the nonphosphorylatable residue alanine (S404A) and the negatively charged amino acid aspartic acid (S404D), respectively. We transfected SynIII KO cortical neurons with either WT SynIII or the Cdk5 phosphorylation mutants of SynIII, as described above for PKA. In primary SynIII KO cortical neurons, the expression and subcellular distribution of both mutants were similar to WT SynIII (Fig. $5 A, B$ ) with overall levels in the cultures that were in the same range of the endogenous SynIII in parallel cultures of WT neurons at the same developmental stage (Fig. 5A). As shown above, expression of WT SynIII fully rescued the KO phenotype. 
Conversely, expression of the nonphosphorylatable S404A SynIII mutant was totally ineffective in correcting the impairment in the length and number of SMI312negative processes, as well as in axon extension and delayed maturation of neurons into SMI312-positive cells (Fig. 5C-E). Interestingly, the pseudo-phosphorylated S404D SynIII mutant, although ineffective in correcting the defective length and number of SMI312-negative processes (Fig. 5D), partially rescued the delayed maturation of neurons into SMI312-positive cells, and fully rescued axonal elongation (Fig. $5 E$ ).

Together, these results indicate that SynIII regulates neurite outgrowth through a Cdk5-dependent phosphorylation, concomitantly or in addition to the PKA-dependent regulation.

\section{Functional SynIII is critical for early neuronal survival}

Neurogenesis consists of a number of stages, including proliferation, survival, and differentiation (Gage, 2000), and previous work reported an effect of SynIII on adult neurogenesis (Kao et al., 2008). Thus, we evaluated cell viability in WT and SynIII KO cortical neurons using the MTT reduction assay (Fig. 6A). The percentage of surviving $\mathrm{KO}$ neurons compared with WT was $73.5 \pm 5.5 \%$ and $57.5 \pm 10.5 \%$ at 3 and 7 DIV, respectively, indicating that SynIII KO cells are significantly less viable than WT neurons (Fig. $6 A)$. This viability phenotype of SynIII $\mathrm{KO}$ neurons at both time windows was fully rescued by the expression of WT SynIII, but not by the expression of PKA or Cdk5 phosphorylation mutants (Fig. 6B). The data demonstrate that the presence of a functional SynIII, which is dynamically phosphorylated by PKA and Cdk5, is crucial for cell survival, and strengthen the knowledge on a key role of PKA and Cdk5 as regulators of neuronal survival (Ohshima et al., 1996; Dagda and Das Banerjee, 2015).

Some of the prominent roles of neurotrophins mediated by TrkB expression and signaling include the regulation of cell survival, neurite outgrowth and branching, and synaptic plasticity (Fenner, 2012). Therefore, we examined the protein expression of full-length TrkB receptor (TrkB-FL) and of its truncated isoform lacking the tyrosine kinase domain (TrkB-T1) in WT and SynIII KO cortical neurons at early stages of development by Western blotting (Fig. 6C). The level of total TrkB, considered as the sum of the full-length and truncated T1 isoforms, rapidly increased during development, reaching a plateau at 5-7 DIV in both WT and $\mathrm{KO}$ neurons, with no significant differences across genotypes (Fig. 6C,D, top). However, we observed a dramatic increase in the TrkB-T1 to TrkB-FL ratio in SynIII KO cells (Fig. $6 C, D)$. Indeed, whereas WT neurons virtually expressed only TrkB-FL at all developmental stages, the expression of TrkB-T1 progressively and significantly increased in SynIII KO neurons, reaching $\sim 50 \%$ of the total TrkB isoforms expressed at 7 DIV (Fig. 6C,D, bottom). The increased expression of TrkB-T1 in
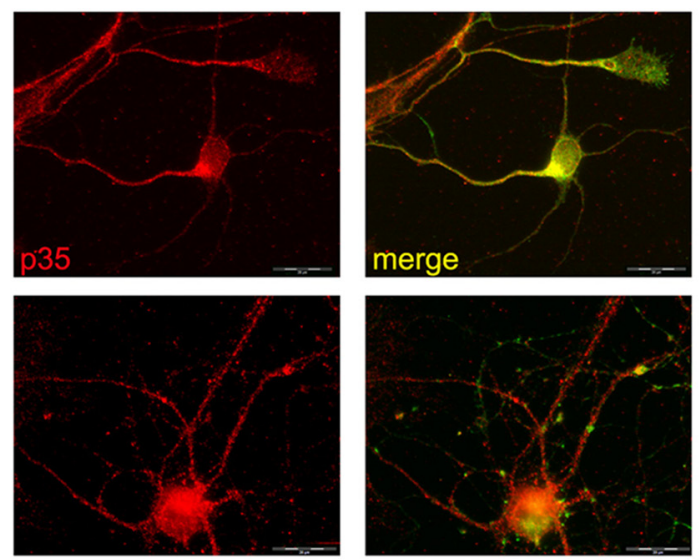

B

Synapsin III cdk5

p35

Synaptophysin

Figure 4. Synlll and Cdk5/p35 subcellular distribution in neurons. $\boldsymbol{A}$, Representative images of WT cortical neurons fixed at 3 and 14 DIV and double stained for SynIII (green) and p35 (red). Scale bar, $20 \mu \mathrm{m}$. B, Subcellular fractions of rat forebrain obtained 列 left in $\mathrm{kDa}$. $\mathrm{H}$, Crude homogenate: S1, postnuclear supernatant: S2, cytosolic and microsomal fraction; P2, crude synaptosomes; gradient-2 fraction, crude synaptic vesicles; SG4, sucrose gradient-4 fraction, synaptic membranes; USV, highly purified synaptic vesicles; SSV, salt-treated synaptic vesicles; FT, flow-through of Controlled-Pore Glass chromatography.

SynIII KO neurons was paralleled by a decrease in TrkB-FL levels. On the other hand, the levels of the $\mathrm{p} 75^{\mathrm{NTR}}$ neurotrophin receptor, a death domain-containing receptor playing complex roles in axon determination, growth, and survival (Yamashita et al., 1999; Ibáñez and Simi, 2012; Zuccaro et al., 2014), was substantially preserved in SynIII KO neurons compared with WT (Fig. 6C; mean \pm SEM percentage p75 ${ }^{\text {NTR }}$ levels in SynIII KO vs WT: $82.19 \pm 15.7, p=0.268$ at $3 \mathrm{DIV} ; 79.19 \pm 13.09, p=0.191$ at 5 DIV; $86.87 \pm 16.9, p=0.176$ at 7 DIV; unpaired Student's $t$ test, $n=4$ ). The reduced survival of SynIII KO neurons (Fig. 6A) prompted us to investigate the expression of caspase 3 , a crucial mediator of programmed cell death, by Western blotting in WT and SynIII KO neuronal lysates (Fig. 6C). Although the levels of endogenous full-length caspase 3 (the $35 \mathrm{kDa}$ inactive zymogen) were not changed in SynIII KO neurons compared with WT (mean \pm SEM percentage full-length caspase3 levels in SynIII KO vs WT: $103.10 \pm 9.13, p=0.968$ at $3 \mathrm{DIV} ; 92.57 \pm 8.63, p=0.930$ at 5 DIV; $86.35 \pm 6.80, p=0.630$ at 7 DIV; unpaired Student's $t$ test, $n=4$ ), the expression level of cleaved caspase 3 (the $17 \mathrm{kDa}$ active subunit) was dramatically increased (Fig. $6 C, E$ ). These effects are likely to be specific to neuronal signaling because astrocytes and microglial cells represent only a negligible percentage of 
A

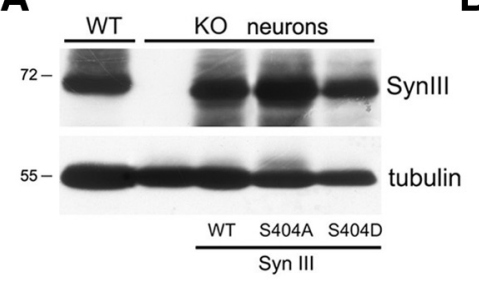

B

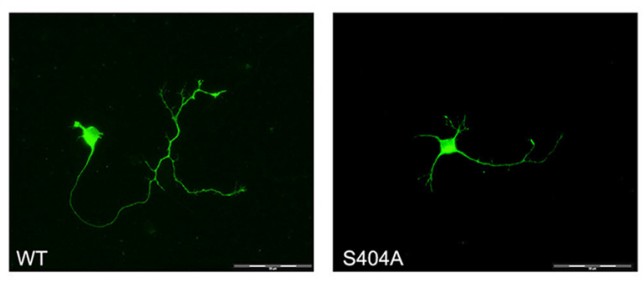

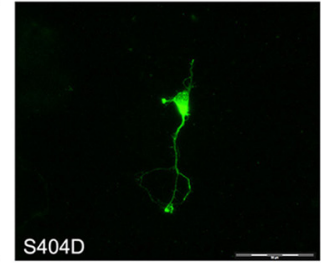

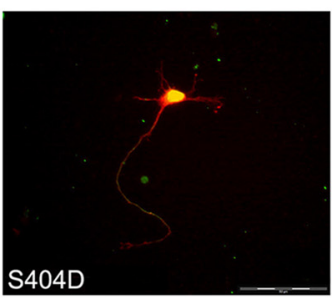

S404A

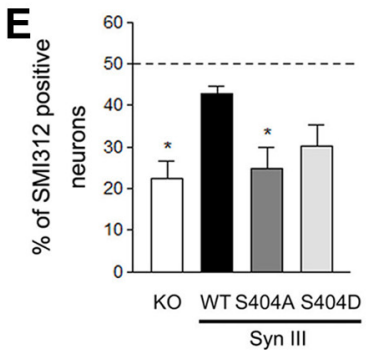

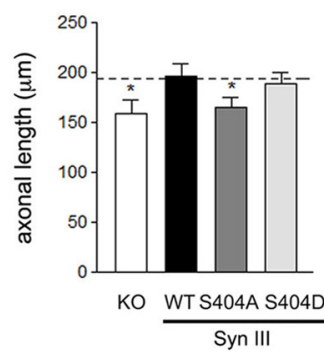

Figure 5. Cdk5 phosphorylation of Synlll regulates the early development of neurons. $\boldsymbol{A}$, Representative Western blot of Synlll expression in WT neurons, K0 neurons, and K0 neurons expressing either WT SynIII or its Cdk5 mutants. Primary cortical neurons from either WT or SynIII K0 mice were transfected by Amaxa before plating with empty pCAGGs-IRES-tdTomato vector as described in the legend to Figure 3 (WT and KO neurons). For rescue experiments, SynIII KO neurons were transfected with pCAGGs-IRES-tdTomato vectors encoding WT-SynIII, S404A-SynIII, or S404D-SynIII. Neurons were lysed at 3 DIV and subjected to Western blotting. Tubulin immunoreactivity was used to control for equal loading. Molecular masses of the proteins are shown on the left in kDa. $\boldsymbol{B}$, Representative images of 3 DIV tdTomato-positive Synlll KO cortical neurons expressing WT-SynIII, S404A-Synlll, or S404D-SynllI. Fixed cells were stained for SynllI (green). WT-Synlll and both mutants showed a similar expression and distribution in all subcellular compartments. Scale bar, $50 \mu \mathrm{m}$. C, Representative images of 3 DIV Synlll K0 cortical neurons expressing tdTomato alone (KO) or with WT-SynIII, S404A-SynIII, or S404D-SynIII (red) and stained for SMI312 (green). Scale bar, $50 \mu \mathrm{m}$. D, Quantification of the length and number of minor processes of neurons in the experiments shown in C measured by ImageJ software. $\boldsymbol{E}$, Quantification of the percentage of SMI312-positive neurons and their axon length. Data are mean \pm SEM ( $n=142 \mathrm{KO}$ neurons, $n=130$ WT-SynllI neurons, $n=116$ S404A-SynllI neurons, and $n=134$ S404D-SynIII neurons at 3 DIV, from $n=4$ independent experiments). ${ }^{*} p<0.05$, versus WT (one-way ANOVA followed by the Bonferroni's multiple-comparison test). ${ }^{* *} p<0.01$, versus WT (one-way ANOVA followed by the Bonferroni's multiple-comparison test). Dashed lines indicate mean values of WT cortical neurons transfected with empty pCAGGs-IRES-tdTomato at 3 DIV included for comparison.

total cells under our culture conditions. Interestingly, a higher degree of apoptosis was present in brain slices from SynIII KO mice (Fig. $6 F$ ). Indeed, we observed a $57.5 \%$ increase of the immunoreactivity of the apoptotic marker active caspase 3 in the neocortex of SynIII KO mice at P7 compared with age-matched WT slices. Together, these results indicate that chronic depletion of functional SynIII leads to reduced cell survival both in vitro and in vivo, and suggest that changes in the complement of TrkB receptor isoforms expressed by neurons activate intracellular apoptotic pathways that may eventually impact on both cell survival and neuronal development.

\section{Discussion}

SynIII is an atypical member of the Syn family. It is concentrated at presynaptic terminals but also distributes in other cellular regions; it is expressed precociously, but its expression declines in the adult (Ferreira et al., 2000; Perlini et al., 2015); its KO in embryonic neurons impairs inhibitory transmission, but its deletion is not epileptogenic (Feng et al., 2002). This intriguing profile could be attributable to the presence of the SynIII-specific domain J, which contains a major phosphorylation site for Cdk5 (Perlini et al., 2015), as well as of domains shared with the other Syns (domains B, C, E, and A, containing a highly conserved phosphorylation site for PKA).

\section{SynIII acts at very early stages of neuronal development}

During early stages in culture, SynIII KO cortical neurons show an immature morphology, with decreased number and length of SMI312-negative processes, delayed axon specification, and impaired axon extension. The phenotype of either SynIII KO neurons or neurons prepared from in utero SynIII-silenced embryos consistently indicates an important role of SynIII in early neuronal development and differentiation (Ferreira et al., 2000; Perlini et al., 2015). Previous studies reported that SynIII knockdown with antisense oligonucleotides or constitutive SynIII KO impaired differentiation and elongation of the axon, with altered growth-cone morphology (Ferreira et al., 2000; Feng et al., 2002). We extended these findings by showing that SynIII plays an important role in the outgrowth of all neuronal processes, as well as in the survival of developing neurons. In addition, our study provides a mechanistic explanation for the defects in migration, orientation, and morphology of cortical pyramidal neurons caused by the acute knockdown of SynIII in vivo (Perlini et al., 2015).

The effects of endogenously expressed SynIII occur in a very narrow temporal window, as demonstrated by the fact that SynIII $\mathrm{KO}$ neurons catch up the growth at later stages in culture and that acute silencing is effective only when SynIII knockdown is performed before plating. However, the expression of exogenous 
A

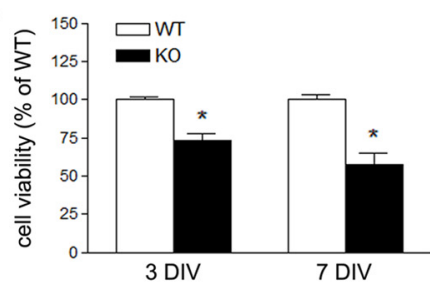

C

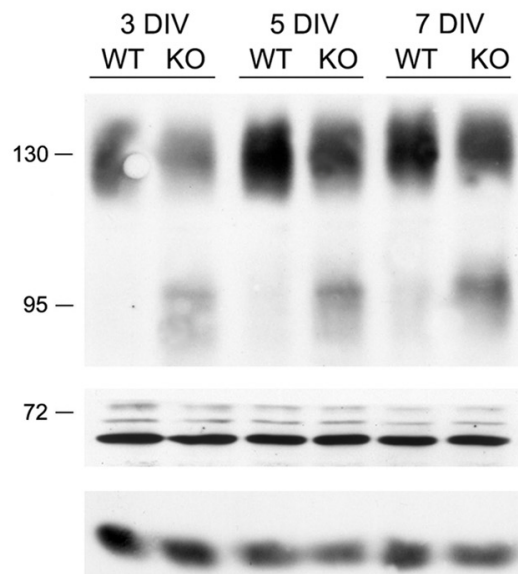

$28-$

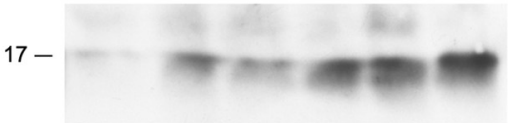

$55-$

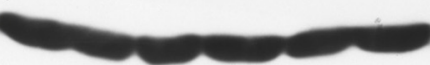

B

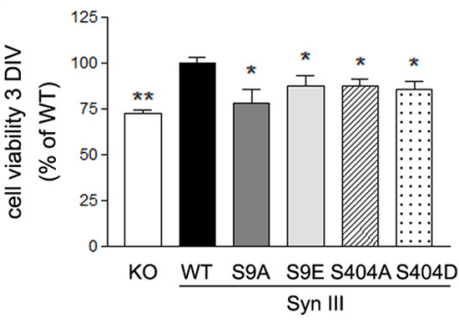

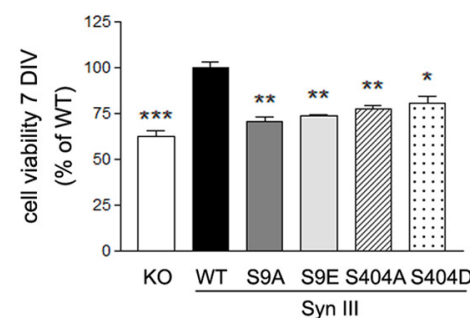

D

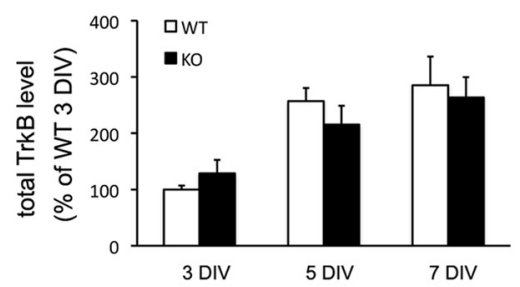

TrkB-T1

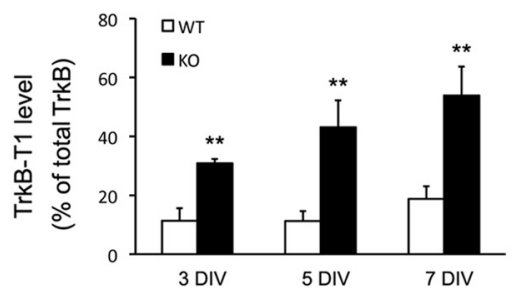

E

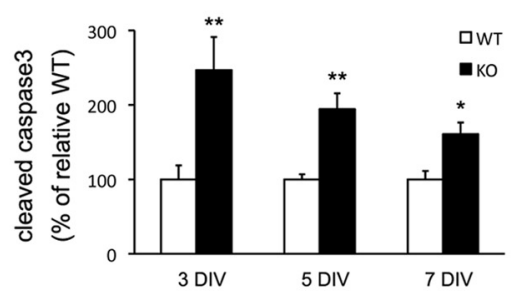

F

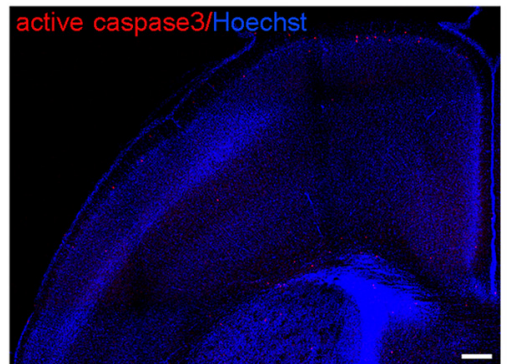

WT

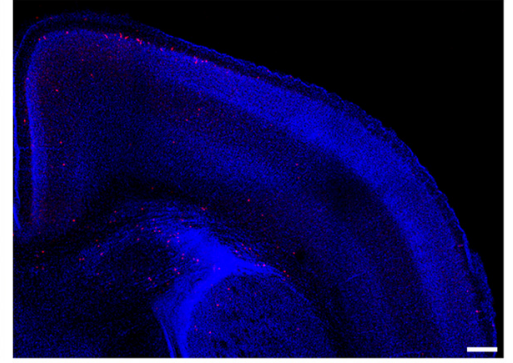

KO

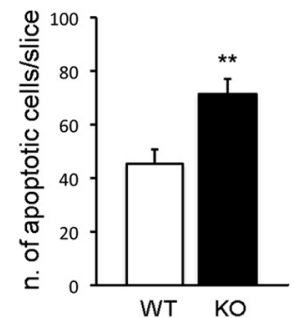

Figure 6. A functional SynIII is critical for early neuron survival. $A$, Cell viability measured by the MTT assay of WT and SynIII KO cortical neurons at 3 and 7 DIV. Data are mean \pm SEM of $n=4$ independent experiments. ${ }^{*} p=0.023$ at 3 DIV (unpaired Student's $t$ test). ${ }^{*} p=0.035$ at 7 DIV (unpaired Student's $t$ test). B, (ell viability evaluated by the MTT assay in SynIII KO cortical neurons transfected before plating with pCAGGs-IRES-tdTomato alone (K0) or with WT-SynIII, S9A-SynIII, S9E-SynIII, S404A-SynllI, and S404D-SynIII at 3 and 7 DIV. Data are mean \pm SEM of $n=4$ independent experiments. ${ }^{*} p<0.05$ versus WT (one-way ANOVA followed by the Bonferroni's multiple-comparison test). ${ }^{* *} p<0.01$ versus WT (one-way ANOVA followed by the Bonferroni's multiple-comparison test). ${ }^{* *} p<0.001$ versus WT (one-way ANOVA followed by the Bonferroni's multiple-comparison test). $C$, Representative Western blots showing the expression level of full-length (TrkB-FL) and truncated (TrkB-T1) TrkB, p75 ${ }^{\text {NTR }}$, caspase3, and cleaved caspase3 in lysates of WT and Synlll K0 cortical neurons at 3, 5 , and 7 DIV. Tubulin immunoreactivity was used to control for equal loading. Molecular masses of the proteins are shown on the left in $\mathrm{kDa}$. The exposures for inactive and active caspase3 shown in the figure were optimized to avoid saturation of the signal in any of the lanes. D, Quantification of the expression level of total TrkB (TrkB-FL plus TrkB-T1, top) and truncated TrkB (TrkB-T1, bottom) in WT and KO cortical neurons at 3, 5, and 7 DIV. Total TrkB is expressed in percentage of the WT level at 3 DIV, whereas truncated TrkB is expressed in percentage of the total TrkB level. Data are mean \pm SEM of $n=4$ independent experiments. ${ }^{* *} p=0.002$ at 3 DIV (unpaired Student's $t$ test). ${ }^{* *} p=0.006$ at 5 and 7 DIV (unpaired Student's $t$ test). $\boldsymbol{E}$, Quantification of the expression level of cleaved capsase3 in WT and KO cortical neurons at 3,5, and 7 DIV expressed in percentage of relative WT level. Data are mean \pm SEM of $n=$ 4 independent experiments. ${ }^{*} p=0.036$ at 7 DIV (unpaired Student's $t$ test). ${ }^{* *} p=0.003$ at 5 DIV (unpaired Student's $t$ test). ${ }^{* *} p=0.007$ at 3 DIV (unpaired Student's $t$ test). $\boldsymbol{F}$, Representative confocal images of active caspase3 immunoreactivity (red) from coronal sections of the neocortex of WT and Synlll KO mouse pups at P7. Slices were counterstained with Hoechst (blue). Scale bar, $200 \mu \mathrm{m}$. Quantification of the number of apoptotic cells located in the cortex per slice in WT and Synlll KO animals is shown on the right. Data are mean \pm SEM of $n=6$ WT mice and $n=5$ Synlll K0 mice. One slice per animal was analyzed. ${ }^{* *} p=0.009$ (unpaired Student's $t$ test). 
SynIII in SynIII KO neurons in vitro can rescue the phenotype at later times. This suggests that SynIII KO neurons remain sensitive to exogenous SynIII, as well as to other compensatory proteins, during a longer temporal window and eventually recover from the phenotype.

Both the delayed migration and the misorientation phenotype observed in vivo could be the consequence of an early defect in process outgrowth and axon determination. It is possible that the developmental effects of SynIII are obtained through interactions with the cytoskeleton, namely, actin and SVs. Indeed, SynIII is present at the growth cone of developing processes, where intense actin remodeling and membrane trafficking take place. Regulation of actin dynamics and SV trafficking are both the bases for elongation of neurites, particularly the axon, and requisites for membrane rearrangements during cell migration along radial glia fibers (Gupta et al., 2003; Itofusa and Kamiguchi, 2011). Dysregulation of SynIII activity could thus interfere with cytoskeletal dynamics and membrane rearrangements, which are essential mechanisms for the outgrowth and elongation of neuritic processes, as well as for migration and correct orientation of the neurons in the cortical layers.

\section{The activity of SynIII in early neuronal development is regulated by PKA and Cdk5 phosphorylation}

We demonstrate that the effects of SynIII on neurite outgrowth and axon specification require PKA- and Cdk5-dependent phosphorylation, as both PKA and Cdk5 dephospho-mimetic mutants were totally unable to rescue the developmental phenotype of SynIII KO neurons. The cAMP-mediated pathway has been implicated in neurite outgrowth, neuronal differentiation, and survival (Kao et al., 2002; Shelly et al., 2010). PKA phosphorylation of SynI regulates SV dynamics at the growth cone in developing neurons (Bonanomi et al., 2005) and the formation of excitatory and inhibitory synapses (Perlini et al., 2011). Phosphorylation of SynII by PKA is involved in precocious stages of neuronal development in Xenopus spinal neurons, where the expression of a pseudo-phosphorylated SynII mutant was shown to stimulate neurite outgrowth and rescue the KO phenotype (Kao et al., 2002). In the case of SynIII, both the dephospho-mimetic and pseudo-phosphorylated SynIII mutants were unable to rescue the neuronal morphology in SynIII KO neurons. This result can be explained by the fact that cycles of phosphorylation/dephosphorylation are required to achieve the SynIII effects and/or by the lack of possible compensatory mechanisms by other Syn isoforms that are poorly expressed at very early stages of development (Ferreira et al., 2000; Perlini et al., 2015).

Cdk5 is a serine/threonine kinase precociously expressed in developing neurons, where it mediates the effects of Sema3a in neuronal growth and differentiation (Nikolic et al., 1996; Ohshima et al., 2007; Yun et al., 2013), as well as in mature neurons, where it regulates SV trafficking at synapses through SynI phosphorylation (Kim and Ryan, 2010; Verstegen et al., 2014). We recently described that SynIII is substrate for Cdk5 at a major site located in the unique domain J (Perlini et al., 2015). The finding that phosphorylation of SynIII has an important functional role in neurite outgrowth, axon specification, and elongation provides a molecular basis for the effects of Cdk5 phosphorylation of SynIII in the radial migration and orientation of pyramidal neurons in vivo (Perlini et al., 2015). Similar to what is observed in cortical development, the C $\mathrm{dk} 5$ phospho-mimetic mutant of SynIII partially rescued the developmental phenotype, whereas the dephospho-mimetic mutant did not. The view of SynIII as a downstream effector of the Cdk5 pathway in the early developmental stage is further strengthened by the following: (1) the correlation between Cdk5 expression, SynIII expression, and phosphorylated SynIII at the Cdk5 site before birth (Perlini et al., 2015); (2) the colocalization between the Cdk5 activator p35 and SynIII at early developmental stages; and (3) the coenrichment of SynIII, Cdk5, and p35 in the same subcellular synaptic fractions of adult brain, where SynIII is highly expressed in immature neurons in neurogenic regions (Pieribone et al., 2002). Because Cdk5 regulates the maturation of newborn granule cells in the adult hippocampus (Jessberger et al., 2008), the latter observation suggests a functional role of SynIII mediated by Cdk5 phosphorylation also in adult neurogenesis.

\section{SynIII enhances the survival of developing neurons}

SynIII is already expressed in nestin-positive neural progenitors, and its depletion affects the proliferation and survival of neural progenitors in the adult dentate gyrus of the hippocampus (Kao et al., 2008). Interestingly, we found that SynIII enhances cell survival after neural precursors have developed into neurons and its genetic deletion increases the number of apoptotic cells in both primary neurons and the neocortex of postnatal mice. The increased apoptosis could be rescued in vitro by WT SynIII, but not by its phosphorylation mutants, indicating that the same cellular mechanisms underlie both the trophic and developmental effects of SynIII. One of these potential mechanisms could be BDNF signaling through the TrkB receptor, an essential modulator of neuronal survival, differentiation, and plasticity. Two major TrkB receptor isoforms exist in the brain: TrkB-FL and C-terminal TrkB-T1, lacking the intracellular tyrosine kinase domain and acting as a dominant-negative BDNF receptor (Klein et al., 1990; Biffo et al., 1995; Eide et al., 1996; Haapasalo et al., 2001). Although the role of TrkB-T1 in cell survival is not completely clarified, neuronal survival is primarily stimulated by TrkB-FL signaling and inhibited by TrkB-T1 (Eide et al., 1996; Ninkina et al., 1996; Haapasalo et al., 2001; Tervonen et al., 2006). TrkB-T1 also participates in neuronal development by regulating distinct modes of dendritic growth or selectively affecting dendrite complexity in certain neuronal populations (Fryer et al., 1997; Yacoubian and Lo, 2000; Carim-Todd et al., 2009). The isoform-specific changes in TrkB expression observed in SynIII KO neurons may therefore reduce the BDNF-induced neuroprotection and/or neurite outgrowth and explain both the decreased cell survival and the impaired neuritic elongation observed in SynIII KO neurons at early stages of development. This phenotype is typical of neurons with defective BDNF signaling (Alcántara et al., 1997; Linnarsson et al., 2000; Cesca et al., 2012). Interestingly, the substantial preservation of the $\mathrm{p} 75^{\mathrm{NTR}}$ neurotrophin receptor in the presence of a defective TrkB signaling may unleash $\mathrm{p} 75^{\mathrm{NTR}}$ to trigger programmed cell death, inhibition of actin dynamics and of neuritic growth (Roux and Barker, 2002; Kaplan and Miller, 2003; Yamashita and Tohyama, 2003). For the first time, our data show that SynIII KO mice have dysregulated TrkB expression, which affects both cell survival and neuronal development and is associated with various neurological and psychiatric disorders (Ferrer et al., 1999; Murer et al., 2001; Castrén, 2014; Fenner et al., 2014).

One interesting issue is how SynIII can be so significant for neuronal development and survival and still the SynIII KO mice have a very mild phenotype. Indeed, we demonstrated that SynIII $\mathrm{KO}$ animals display an altered cortical cytoarchitecture similar to that observed in ShRNA-transfected brains (Perlini et al., 2015) with increased apoptosis of neocortical neurons (this paper). Thus, the gross brain morphology is only "apparently normal" 
because previous studies did not use techniques suitable to detect this kind of phenotype.

In conclusion, we found that SynIII plays a central role in early stages of neuronal development involving phosphorylationdependent neuronal survival, polarization, and neuritic growth. These results possibly explain the recently described neurodevelopmental defects in the migration and orientation of SynIIIdepleted cortical neurons (Perlini et al., 2015). Furthermore, these data support the possibility of an association of SynIII with neurodevelopmental disorders, such as schizophrenia, as proposed on the basis of postmortem (Vawter et al., 2002; Porton and Wetsel, 2007) and genetic studies (Porton et al., 2004; Lachman et al., 2005; Chen et al., 2009).

\section{References}

Alcántara S, Frisén J, del Rio JA, Soriano E, Barbacid M, Silos-Santiago I (1997) TrkB signaling is required for postnatal survival of CNS neurons and protects hippocampal and motor neurons from axotomy-induced cell death. J Neurosci 17:3623-3633. Medline

Biffo S, Offenhäuser N, Carter BD, Barde YA (1995) Selective binding and internalisation by truncated receptors restrict the availability of BDNF during development. Development 121:2461-2470. Medline

Bonanomi D, Menegon A, Miccio A, Ferrari G, Corradi A, Kao HT, Benfenati F, Valtorta F (2005) Phosphorylation of synapsin I by cAMP-dependent protein kinase controls synaptic vesicle dynamics in developing neurons. J Neurosci 25:7299-7308. CrossRef Medline

Brewer GJ, Torricelli JR, Evege EK, Price PJ (1993) Optimized survival of hippocampal neurons in B27-supplemented Neurobasal, a new serumfree medium combination. J Neurosci Res 135:567-576. Medline

Carim-Todd L, Bath KG, Fulgenzi G, Yanpallewar S, Jing D, Barrick CA, Becker J, Buckley H, Dorsey SG, Lee FS, Tessarollo L (2009) Endogenous truncated TrkB.T1 receptor regulates neuronal complexity and TrkB kinase receptor function in vivo. J Neurosci 29:678-685. CrossRef Medline

Castrén E (2014) Neurotrophins and psychiatric disorders. Handb Exp Pharmacol 220:461-479. CrossRef Medline

Cesca F, Baldelli P, Valtorta F, Benfenati F (2010) The synapsins: key actors of synapse function and plasticity. Prog Neurobiol 91:313-348. CrossRef Medline

Cesca F, Yabe A, Spencer-Dene B, Scholz-Starke J, Medrihan L, Maden CH, Gerhardt H, Orriss IR, Baldelli P, Al-Qatari M, Koltzenburg M, Adams RH, Benfenati F, Schiavo G (2012) Kidins220/ARMS mediates the integration of the neurotrophin and VEGF pathways in the vascular and nervous systems. Cell Death Differ 19:194-208. CrossRef Medline

Chen Q, Che R, Wang X, O'Neill FA, Walsh D, Tang W, Shi Y, He L, Kendler KS, Chen X (2009) Association and expression study of synapsin III and schizophrenia. Neurosci Lett 465:248-251. CrossRef Medline

Dagda RK, Das Banerjee T (2015) Role of protein kinase A in regulating mitochondrial function and neuronal development: implications to neurodegenerative diseases. Rev Neurosci 26:359-370. CrossRef Medline

dal Maschio M, Ghezzi D, Bony G, Alabastri A, Deidda G, Brondi M, Sato SS, Zaccaria RP, Di Fabrizio E, Ratto GM, Cancedda L (2012) High-performance and sitedirected in utero electroporation by a triple-electrode probe. Nat Commun 3:960. CrossRef Medline

Eide FF, Vining ER, Eide BL, Zang K, Wang XY, Reichardt LF (1996) Naturally occurring truncated trkB receptors have dominant inhibitory effects on brain-derived neurotrophic factor signaling. J Neurosci 16:3123-3129. Medline

Elbashir SM, Harborth J, Lendeckel W, Yalcin A, Weber K, Tuschl T (2001) Duplexes of 21-nucleotide RNAs mediate RNA interference in cultured mammalian cells. Nature 411:494-498. CrossRef Medline

Feng J, Chi P, Blanpied TA, Xu Y, Magarinos AM, Ferreira A, Takahashi RH, Kao HT, McEwen BS, Ryan TA, Augustine GJ, Greengard P (2002) Regulation of neurotransmitter release by synapsin III. J Neurosci 22:43724380. Medline

Fenner BM (2012) Truncated TrkB: beyond a dominant negative receptor. Cytokine Growth Factor Rev 23:15-24. CrossRef Medline

Fenner ME, Achim CL, Fenner BM (2014) Expression of full-length and truncated trkB in human striatum and substantia nigra neurons: implications for Parkinson's disease. J Mol Histol 45:349-361. CrossRef Medline
Ferreira A, Kao HT, Feng J, Rapoport M, Greengard P (2000) Synapsin III: developmental expression, subcellular localization, and role in axon formation. J Neurosci 20:3736-3744. Medline

Ferrer I, Marín C, Rey MJ, Ribalta T, Goutan E, Blanco R, Tolosa E, Martí E (1999) BDNF and full-length and truncated TrkB expression in Alzheimer disease: implications in therapeutic strategies. J Neuropathol Exp Neurol 58:729-739. CrossRef Medline

Fornasiero EF, Bonanomi D, Benfenati F, Valtorta F (2010) The role of synapsins in neuronal development. Cell Mol Life Sci 67:1383-1396. CrossRef Medline

Fornasiero EF, Raimondi A, Guarnieri FC, Orlando M, Fesce R, Benfenati F, Valtorta F (2012) Synapsins contribute to the dynamic spatial organization of synaptic vesicles in an activity-dependent manner. J Neurosci 32:12214-12227. CrossRef Medline

Fryer RH, Kaplan DR, Kromer LF (1997) Truncated trkB receptors on nonneuronal cells inhibit BDNF-induced neurite outgrowth in vitro. Exp Neurol 148:616-627. CrossRef Medline

Gage FH (2000) Mammalian neural stem cells. Science 287:1443-1448.

Gupta A, Sanada K, Miyamoto DT, Rovelstad S, Nadarajah B, Pearlman AL, Brunstrom J, Tsai LH (2003) Layering defect in p35 deficiency is linked to improper neuronal-glial interaction in radial migration. Nat Neurosci 6:1284-1291. CrossRef Medline

Haapasalo A, Koponen E, Hoppe E, Wong G, Castrén E (2001) Truncated trkB.T1 is dominant negative inhibitor of trkB.TK ${ }^{+}$-mediated cell survival. Biochem Biophys Res Commun 280:1352-1358. CrossRef Medline

Hayashi K, Kawai-Hirai R, Harada A, Takata K (2003) Inhibitory neurons from fetal rat cerebral cortex exert delayed axon formation and active migration in vitro. J Cell Sci 116:4419-4428. CrossRef Medline

Huttner WB, Schiebler W, Greengard P, De Camilli P (1983) Synapsin I (protein I), a nerve terminal-specific phosphoprotein: III. Its association with synaptic vesicles studied in a highly purified synaptic vesicle preparation. J Cell Biol 96:1374-1388. CrossRef Medline

Ibáñez CF, Simi A (2012) p75 neurotrophin receptor signaling in nervous system injury and degeneration: paradox and opportunity. Trends Neurosci 35:431-440. CrossRef Medline

Itofusa R, Kamiguchi H (2011) Polarizing membrane dynamics and adhesion for growth cone navigation. Mol Cell Neurosci 48:332-338. CrossRef Medline

Jessberger S, Aigner S, Clemenson GD Jr, Toni N, Lie DC, Karalay O, Overall R, Kempermann G, Gage FH (2008) Cdk5 regulates accurate maturation of newborn granule cells in the adult hippocampus. PLoS Biol 6:e272. CrossRef Medline

Jovanovic JN, Benfenati F, Siow YL, Sihra TS, Sanghera JS, Pelech SL, Greengard P, Czernik AJ (1996) Neurotrophins stimulate phosphorylation of synapsin I by MAP kinase and regulate synapsin I-actin interactions. Proc Natl Acad Sci U S A 93:3679-3683. CrossRef Medline

Kao HT, Porton B, Czernik AJ, Feng J, Yiu G, Häring M, Benfenati F, Greengard P (1998) A third member of the synapsin gene family. Proc Natl Acad Sci U S A 95:4667-4672. CrossRef Medline

Kao HT, Porton B, Hilfiker S, Stefani G, Pieribone VA, DeSalle R, Greengard P (1999) Molecular evolution of the synapsin gene family. J Exp Zool 285:360-377. CrossRef Medline

Kao HT, Song HJ, Porton B, Ming GL, Hoh J, Abraham M, Czernik AJ, Pieribone VA, Poo MM, Greengard P (2002) A protein kinase A-dependent molecular switch in synapsins regulates neurite outgrowth. Nat Neurosci 5:431-437. CrossRef Medline

Kao HT, Li P, Chao HM, Janoschka S, Pham K, Feng J, Mcewen BS, Greengard P, Pieribone VA, Porton B (2008) Early involment of synapsin III in neural progenitor cell development in the adult hippocampus. J Comp Neurol 507:1860-1870. CrossRef Medline

Kaplan DR, Miller FD (2003) Axon growth inhibition: signals from the p75 neurotrophin receptor. Nat Neurosci 6:435-436. CrossRef Medline

Kim SH, Ryan TA (2010) CDK5 serves as a major control point in neurotransmitter release. Neuron 67:797-809. CrossRef Medline

Klein R, Conway D, Parada LF, Barbacid M (1990) The trkB tyrosine protein kinase gene codes for a second neurogenic receptor that lacks the catalytic kinase domain. Cell 61:647-656. CrossRef Medline

Lachman HM, Stopkova P, Rafael MA, Saito T (2005) Association of schizophrenia in African Americans to polymorphism in synapsin III gene. Psychiatr Genet 15:127-132. CrossRef Medline

Linnarsson S, Willson CA, Ernfors P (2000) Cell death in regenerating pop- 
ulations of neurons in BDNF mutant mice. Brain Res Mol Brain Res 75:61-69. CrossRef Medline

Matsubara M, Kusubata M, Ishiguro K, Uchida T, Titani K, Taniguchi H (1996) Site-specific phosphorylation of synapsin I by mitogen-activated protein kinase and Cdk5 and its effects on physiological functions. J Biol Chem 271:21108-21113. CrossRef Medline

Murer MG, Yan Q, Raisman-Vozari R (2001) Brain-derived neurotrophic factor in the control human brain, and in Alzheimer's disease and Parkinson's disease. Prog Neurobiol 63:71-124. CrossRef Medline

Nikolic M, Dudek H, Kwon YT, Ramos YF, Tsai LH (1996) The ckd5/p35 kinase is essential for neurite outgrowth during neuronal differentiation. Genes Dev 10:816-825. CrossRef Medline

Ninkina N, Adu J, Fischer A, Piñón LG, Buchman VL, Davies AM (1996) Expression and function of TrkB variants in developing sensory neurons. EMBO J 15:6385-6393. Medline

Odajima J, Wills ZP, Ndassa YM, Terunuma M, Kretschmannova K, Deeb TZ, Geng Y, Gawrzak S, Quadros IM, Newman J, Das M, Jecrois ME, Yu Q, Li N, Bienvenu F, Moss SJ, Greenberg ME, Marto JA, Sicinski P (2011) Cyclin E constrains Cdk5 activity to regulate synaptic plasticity and memory formation. Dev Cell 21:655-668. CrossRef Medline

Ohshima T, Ward JM, Huh CG, Longenecker G, Veeranna A, Pant HC, Brady RO, Martin LJ, Kulkarni AB (1996) Targeted disruption of the cyclindependent kinase 5 gene results in abnormal corticogenesis, neuronal pathology and perinatal death. Proc Natl Acad Sci U S A 93:11173-11178. CrossRef Medline

Ohshima T, Hirasawa M, Tabata H, Mutoh T, Adachi T, Suzuki H, Saruta K, Iwasato T, Itohara S, Hashimoto M, Nakajima K, Ogawa M, Kulkarni AB, Mikoshiba K (2007) Cdk5 is required for multipolar-to-bipolar transition during radial neuronal migration and proper dendrite development of pyramidal neurons in the cerebral cortex. Development 134:22732282. CrossRef Medline

Orenbuch A, Shalev L, Marra V, Sinai I, Lavy Y, Kahn J, Burden JJ, Staras K, Gitler D (2012) Synapsin selectively controls the mobility of resting pool vesicles at hippocampal terminals. J Neurosci 32:3969-3980. CrossRef Medline

Orlando M, Lignani G, Maragliano L, Fassio A, Onofri F, Baldelli P, Giovedí S, Benfenati F (2014) Functional role of ATP binding to synapsin I in synaptic vesicle trafficking and release dynamics. J Neurosci 34:1475214768. CrossRef Medline

Perlini LE, Botti F, Fornasiero EF, Giannandrea M, Bonanomi D, Amendola M, Naldini L, Benfenati F, Valtorta F (2011) Effects of phosphorylation and neuronal activity of synapse formation by synapsin I. J Cell Sci 124: 3643-3653. CrossRef Medline

Perlini LE, Szczurkowska J, Ballif BA, Piccini A, Sacchetti S, Giovedì S, Benfenati F, Cancedda L (2015) Synapsin III acts downstream of semaphorin $3 \mathrm{~A} / \mathrm{CDK} 5$ signaling to regulate radial migration and orientation of pyramidal neurons in vivo. Cell Rep 11:234-248. CrossRef Medline

Pieribone VA, Porton B, Rendon B, Feng J, Greengard P, Kao HT (2002) Expression of synapsin III in nerve terminals and neurogenic regions of the adult brain. J Comp Neurol 454:105-114. CrossRef Medline

Porton B, Wetsel WC (2007) Reduction of synapsin III in the prefrontal cortex of individuals with schizophrenia. Schizophr Res 94:366-370. CrossRef Medline
Porton B, Kao HT, Greengard P (1999) Characterization of transcripts from the synapsin III gene locus. J Neurochem 73:2266-2271. Medline

Porton B, Ferreira A, DeLisi LE, Kao HT (2004) A rare polymorphism affects a mitogen-activated protein kinase site in synapsin III: possible relationship to schizophrenia. Biol Psychiatry 55:118-125. CrossRef Medline

Porton B, Rodriguiz RM, Phillips LE, Gilbert JW 4th, Feng J, Greengard P, Kao HT, Wetsel WC (2010) Mice lacking synapsin III show abnormalities in explicit memory and conditioned fear. Genes Brain Behav 9:257268. CrossRef Medline

Roux PP, Barker PA (2002) Neurotrophin signaling through the p75 neurotrophin receptor. Prog Neurobiol 67:203-233. CrossRef Medline

Shelly M, Lim BK, Cancedda L, Heilshorn SC, Gao H, Poo MM (2010) Local and long-range reciprocal regulation of cAMP and cGMP in axon/dendrite formation. Science 327:547-552. CrossRef Medline

Stichel CC, Muller HW (1991) Dissociated cell culture of rat cerebral cortical neurons in serum-free, conditioned media: GABA-immunopositive neurons. Dev Brain Res 64:145-154. CrossRef Medline

Südhof TC, Czernik AJ, Kao HT, Takei K, Johnston PA, Horiuchi A, Wagner MA, Kanazir SD, Perin MS, De Camilli P, et al. (1989) Synapsins: mosaic of shared and individual domains in a family of synaptic vesicle phosphoproteins. Science 245:1474-1480. CrossRef Medline

Tervonen TA, Ajamian F, De Wit J, Verhaagen J, Castrén E, Castrén M (2006) Overexpression of a truncated TrkB isoform increases the proliferation of neural progenitors. Eur J Neurosci 24:1277-1285. CrossRef

Valtorta F, Greengard P, Fesce R, Chieregatti E, Benfenati F (1992) Effects of the neuronal phosphoprotein synapsin I on actin polymerization: I. Evidence for a phosphorylation-dependent nucleating effect. J Biol Chem 267:11281-11288. Medline

Vawter MP, Thatcher L, Usen N, Hyde TM, Kleinman JE, Freed WJ (2002) Reduction of synapsin in the hippocampus of patients with bipolar disorder and schizophrenia. Mol Psychiatry 7:571-578. CrossRef Medline

Verstegen AM, Tagliatti E, Lignani G, Marte A, Stolero T, Atias M, Corradi A, Valtorta F, Gitler D, Onofri F, Fassio A, Benfenati F (2014) Phosphorylation of synapsin I by cyclin-dependent kinase- 5 sets the ratio between the resting and recycling pools of synaptic vesicles at hippocampal synapses. J Neurosci 34:7266-7280. CrossRef Medline

Yacoubian TA, Lo DC (2000) Truncated and full-length TrkB receptors regulate distinct modes of dendritic growth. Nat Neurosci 3:342-349. CrossRef Medline

Yamashita T, Tohyama M (2003) The p75 receptor acts as a displacement factor that releases Rho from Rho-GDI. Nat Neurosci 6:461-467. CrossRef Medline

Yamashita T, Tucker KL, Barde YA (1999) Neurotrophin binding to the p75 receptor modulates Rho activity and axonal outgrowth. Neuron 24:585593. CrossRef Medline

Yun J, Nagai T, Furukawa-Hibi Y, Kuroda K, Kaibuchi K, Greenberg ME, Yamada K (2013) Neuronal Pern Arnt Sim (PAS) domain protein 4 (NPAS4) regulates neurite outgrowth and phosphorylation of synapsin I. J Biol Chem 288:2655-2664. CrossRef Medline

Zuccaro E, Bergami M, Vignoli B, Bony G, Pierchala BA, Santi S, Cancedda L, Canossa M (2014) Polarized expression of p75(NTR) specifies axons during development and adult neurogenesis. Cell Rep 7:138-152. CrossRef Medline 